\section{Distribution of Radio-Frequency Brightness across the Solar Disk and the Derivation of a Model Corona}

Determinations of the distribution of radiofrequency brightness across the solar disk have been made by Stanier ${ }^{1}$ at a wave-length of $60 \mathrm{~cm}$. and by Machin $^{2}$ at a wave-length of 3.7 metres. A series of similar observations has been carried out by one of $u^{3}$ at wave-lengths of $1 \cdot 4,3 \cdot 7$ and 7.9 metres during the period July 1951-July 1952. In this communication we describe the results of an attempt to derive a model of the corona which is consistent with these observations ${ }^{4}$. made about $N$ or $T$; instead, an attempt was made to derive these quantities directly from the radio observations. The model which was found to fit the observations most closely was given by :

$$
\begin{aligned}
& N=1.6 \times 10^{8} R^{-5} \mathrm{~cm} \cdot .^{-8} . \\
& T=2 \times 10^{8} R^{-3} \text { deg. K. }
\end{aligned}
$$

over the range $R=1 \cdot 2 R_{0}$ to $3 \cdot 0 R_{0}$, where $R_{0}$ is the radius of the photosphere.

This distribution of electron density agrees well with that of Allen for small values of $R$, but for $R$ greater than $2 R_{0}$ the density decreases more slowly outwards.

The extent to which the new model can account for the observations is shown in the graphs. It is

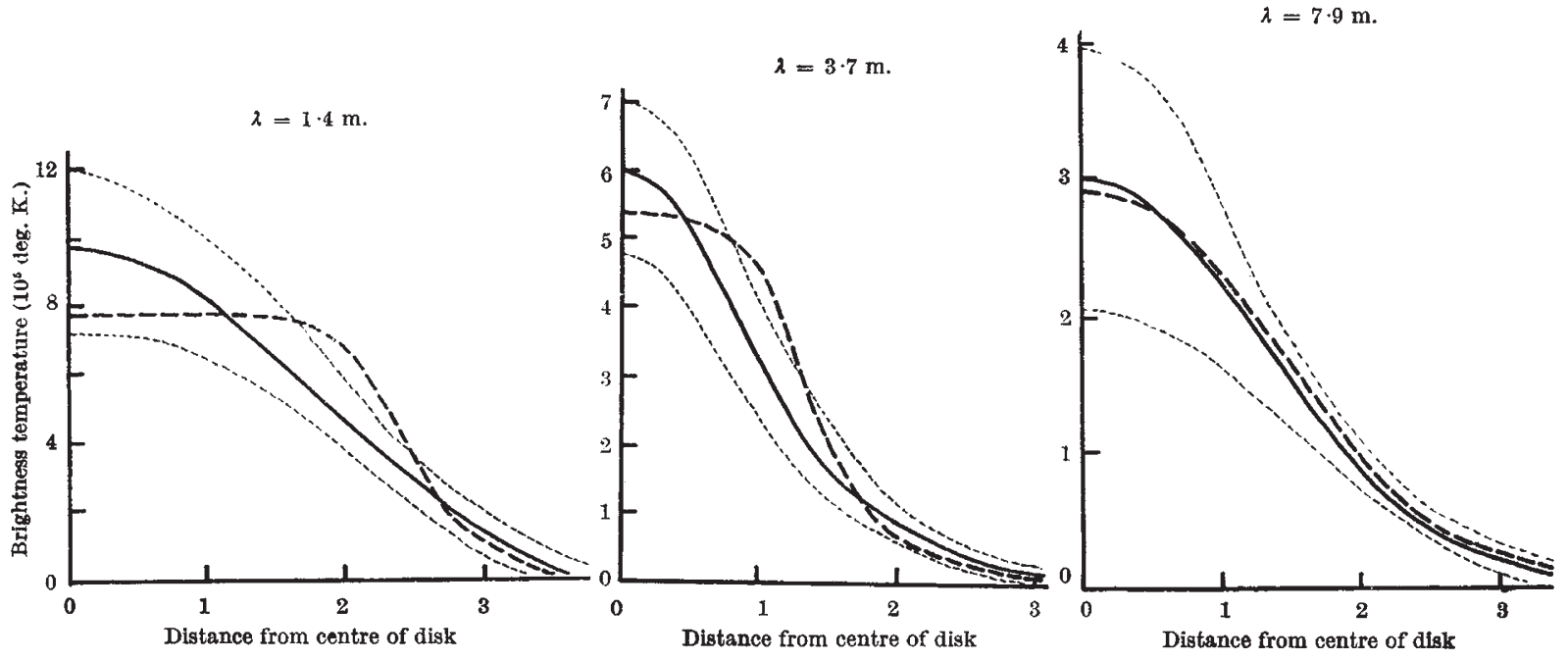

Distribution of radio brightness across the solar disk at three wave-lengths. The distance from the centre of the disk is given in terms of the radius of the photosphere $\left(R_{0}\right)$.

- Experimental curves; -..--.--, limits within which the experimental curves are believed to fall ; - - - theoretical curves

The experiment showed that the contours of constant radio brightness were roughly elliptical in form and varied slightly from day to day. As a first approximation for the purposes of theoretical interpretation, they were replaced by constant circles. The average radial distributions which were used in this way to represent the experimental results are shown as continuous lines in the accompanying graphs. The uncertainty of the experimental results is indicated by the dotted lines, which show the limits within which the distributions are believed to fall. The limits are predominantly caused by an uncertainty in the absolute scale of brightness (because small errors in the distribution for large radii cen introduce large errors in the determination of the central temperature). There is also some uncertainty in the results arising from the day-to-day variations.

Smerd $^{5}$ has derived theoretical curves for the distribution of brightness for a number of wavelengths, assuming the coronal electron density, $N$, given by Allen ${ }^{6}$ and a number of uniform tem. peratures, $T$. It is clear from a comparison of the observations with these theoretical curves that a coronal model with uniform temperature cannot account for the observations, and, in particular, that it cannot provide sufficient intensity at large radii.

If the electron density derived by Allen is retained, analysis showed that these discrepancies could not be removed by assuming that the temperature varied with height in the corona. A new method of analysis was therefore adopted in which no assumptions were seen that a satisfactory fit is obtained for $\rho>1.5 R$. (where $p$ is the distance from the centre of the disk), which is the important region where Smerd's curves show the greatest discrepancy with observation. For values of $p$ between $0.5 R_{0}$ and $1.5 R_{0}$ the intensity at 1.4 and 3.7 metres is somewhat greater than that observed. It seems likely, however, that this remaining discrepancy may be removed when it has been possible to allow for the effects of scattering by irregularities of electron density in the corona. Some information about these irregularities for $R>5 R$. has recently been obtained by Hewish? from observations of the occultation by the solar corona of the intense radio source in Taurus. It is possible that the inclusion of the scattering effects in the analysis may result in some modification to the values of $N$ and $T$ derived above.

This work was carried out as part of a programme of radio research at the Cavendish Laboratory, supported by the Department of Scientific and Industrial Research.

\section{P. A. O'BrIEN}

C. J. BELT.

Cavendish Laboratory,

Cambridge. Dec. 14.

${ }^{1}$ Stanier, H. M., Nature, 165, 354 (1950).

Machin, K. E., Nature, 167, 889 (1951).

${ }^{8}$ O'Brien, P. A., Mon. Not. Roy. Astro. Soc., 113, No. 5 (1953).

4 Ryle, M., Volta Convention Proc., Accademia Nazionale del Lincei, 276 (1952)

s Smerd, S. F., Aust. J. Sci. Res., 3 A, 34 (1950).

Allen, C. W., Mon. Not. Roy. Astro. Soc., 107, 426 (1947)

'Allen, C. W., Mon. Not. Ro
${ }^{7}$ Hewish, A. (in preparation). 